\title{
Successful endovascular treatment of ruptured giant aneurysm of arteria lusoria
}

\author{
Krzysztof Pyra, Maciej Szmygin, Michał Sojka, Tomasz Jargiełto \\ Department of Interventional Radiology and Neuroradiology, Medical University of Lublin, Poland
}

Adv Interv Cardiol 2020; 16, 3 (61): 356-357

DOI: https://doi.org/10.5114/aic.2020.99279

An aberrant right subclavian artery (ARSA - arteria lusoria) is one of the most common congenital anomalies of the aortic arch. The majority of patients remain asymptomatic and the findings are incidental [1]. However, depending on the location and the course of ARSA, degree of compression of mediastinal organs and concomitant vascular anomalies it may manifest with dyspnea, dysphagia or chronic cough. We present a patient with ruptured aneurysmal ARSA managed by endovascular means.

A 73-year old male patient was admitted to the Emergency Department reporting dyspnea, dysphagia and chest pain. Physical examination revealed auscultatory diminished breath sounds over right lung and tachycardia. Lab tests depicted anemia: red blood cells - $2.98 \times 10^{12}$, hemoglobin (HGB) - 9.6 g/dl. Computed tomography angiography (CT-angio) revealed giant saccular aneurysm of ARSA with heterogeneous thrombotic material and contrast enhancement inside the aneurysm sac, reduced cardiac silhouette and hemothorax of the right lung (Figures $1 \mathrm{~A}, \mathrm{~B}$ ). Due to his life-threatening condition the patient was qualified for treatment. He gave informed refusal for open surgery and was treated with endovascular means only.

In general anesthesia both right femoral and left brachial arteries were punctured and vascular sheaths introduced. Cerebral angiography was performed. It revealed severe proximal stenosis of the right internal carotid artery (RICA) and a fully patent circle of Willis. From the groin access the right subclavian artery was catheterized and primary embolization performed with a $14 \mathrm{~mm} \mathrm{Am-}$ platzer Vascular Plug (St. Jude Medical, MN, USA). Afterwards, a cTAG GORE $30 \times 150 \mathrm{~mm}$ stent graft (GORE, DE, USA) was introduced and implanted in the aortic arch and descending aorta covering the ARSA and left subclavian artery. Via axillary access a chimney stent was deployed in order to ensure blood flow to the left vertebral and brachial artery. Embolization of the aneurysmal sac was performed with coils - MReye and Nester (Cook Medical, IN, USA). Finally, balloon angioplasty and stenting of RICA were performed. Control angiography revealed proper stent placement with no filling of the aneurysmal sac and intended left-to-right steal syndrome. Due to the weak arterial pulse of the left upper extremity additional stents were placed into the chimney stent (Figure $1 \mathrm{C}$ ).

After the procedure, the patient remained in a stable clinical condition. Control CT-angio showed no filling of the aneurysm and proper blood flow in stented vessels (Figure 1 D). After 14 days of hospitalization he was discharged in good clinical condition.

First successful repair of ARSA involving thoracotomy and ligation of the artery was described by Gross in 1946 [2]. Since then, many surgical approaches have been applied with positive results but a significant mortality rate [3]. Therefore, hybrid and endovascular techniques were introduced. The most common include stent graft implantation with surgical revascularization (bypass, vessel debranching or transposition). Our patient refused surgery and was treated by endovascular means in an emergency setting. This approach increases the risk of ischemic complications (limb ischemia, stroke) but potential benefits outweigh the risks. In such circumstances anatomic compatibility of ARSA, careful selection of endovascular devices and well-planned treatment are crucial in order to avoid possible intra-procedural and long-term complications. Jalaie et al. described a case of a patient treated similarly by endovascular techniques only with no evidence of limb ischemia or stroke in long-term follow-up [4]. Coil embolization raises the concern of persistent compression of the esophagus as the regression of the aneurysm takes time. However, the patient is regularly seen in the outpatient clinic and does not report dysphagia.

In conclusion, we believe that the described case adds to the limited number of reports of successful endovascular treatment of ARSA aneurysm.

\section{Corresponding author:}

Dr. Maciej Szmygin, Department of Interventional Radiology and Neuroradiology, Medical University of Lublin, Lublin, Poland,

e-mail: mszmygin@gmail.com

Received: 31.03.2020, accepted: 25.04.2020. 

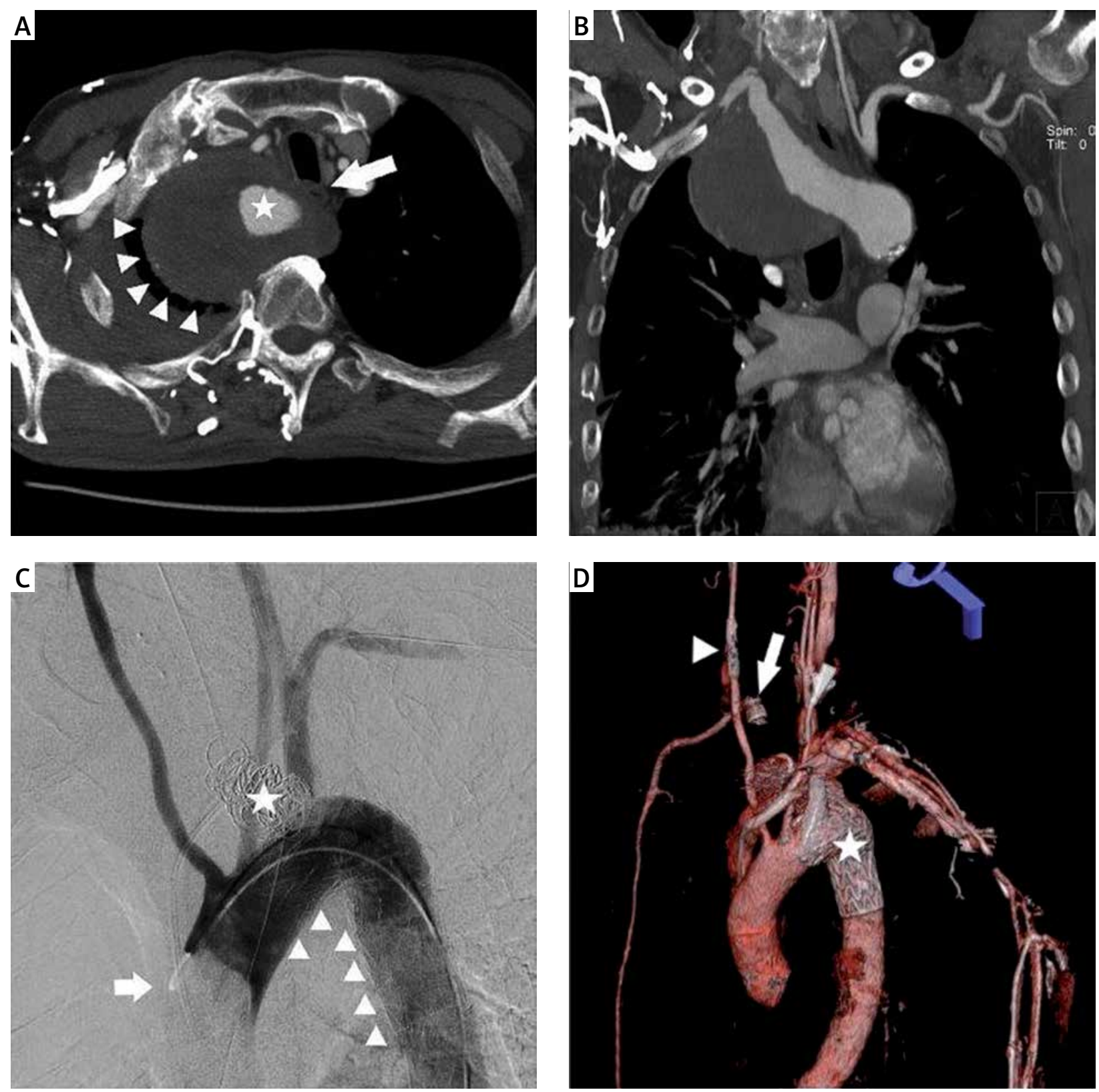

Figure 1. A, B - Transverse and coronal angio-CT scan revealing giant aneurysm (white triangles) of arteria lusoria (white star). White arrow shows compression of esophagus caused by aneurysmal sac. C -Endovascular procedure: control angiography performed from diagnostic catheter (white arrow) presenting vascular plug in the ARSA aneurysmal sac below the origin of the right vertebral artery (first plug plate located distally and the rest proximally to the vessel angulation which ensured a stable position - white star) and cTAG GORE stent graft (white triangles). D - Post-procedural control angio-CT. 3D reconstruction of the aorta and aortic branches confirming successful outcome of the procedure. Visible vascular plug (white arrow), carotid stent (white triangle) and stent graft (white star). Right upper extremity supply from subclavian steal syndrome

\section{Conflict of interest}

The authors declare no conflict of interest.

\section{References}

1. Rouman M, Petrovitch A, Gey EM, Kuntze T. Interdisciplinary management of a perforated aneurysmal arteria lusoria: a case report. Thorac Cardiovasc Surg Rep 2017; 6: e15-7.
2. Gross RE. Surgical treatment for dysphagia lusoria. Ann Surg 1946; 124: 532-4.

3. Kieffer E, Bahnini A, Koskas F. Aberrant subclavian artery: surgical treatment in thirty-three adult patients. J Vasc Surg 1994; 19: 100e9.

4. Jalaie H, Grommes J, Sailer A, et al. Treatment of symptomatic aberrant subclavian arteries. Eur J Vasc Endovasc Surg 2014; 48: 521-6. 